\title{
In situ High Resolution Electron Microscopy
}

\author{
H.W. Zandbergen, B. Song, M. Rudneva and M.A. van Huis \\ Kavli Institute of Nanoscience, Delft University of Technology \\ Lorentzweg 1, 2628 CJ Delft, The Netherlands
}

The recent improvements in the optical performance of high resolution electron microscopes allows for two big steps: a) 3D analysis on the atomic scale of materials (like elemental composition at an interface including the location of doping atoms) and b) in-situ observations of changes in a material driven by an added force, like an electrical current, a temperature change or even the electron beam. In Delft we choose the second topic as our main research theme, in part because we have large expertise on developing TEM sample holders, which is essential for the development of in-situ HREM.

$100 \mathrm{Au}$ surfaces have been studied in profile imaging at room temperature and $\sim 100 \mathrm{~K}$. In both cases the atoms on the surfaces show a high mobility, in part kicked around by the electron beam. These atom movements are in agreement with molecular dynamics simulations. At room temperature we frequently observe that a small island on the top of a hillock is injected into the lower lattice plate thus forming a surface dislocation (See figure 1). The surface dislocation moves around for some time (seconds to minutes) and is then squeezed out along the electron beam direction. At $\sim 100 \mathrm{~K}$ the formation of such a surface dislocation is relatively rare, but the mobility of the Au atoms of the surface is still very high, in some cases even much higher than at room temperature.

HREM was at done on small $\mathrm{Au}$ particles in the temperature range 500 -800 C (see Figure 2). The figure shows many different shapes of a $\mathrm{Au}$ particle that sits on the edge of a SiN membrane in which a hole was formed by the electron beam. The membrane is part of a MEMS based heater, which allows for sub $1 \AA$ resolution at temperatures up to $800 \mathrm{C}$. We are not getting sub $1 \AA$ resolution images at such temperatures routinely.

Recently we have realized a liquid nitrogen cooling holder with 8 electric feed-throughs that allow for the in-situ study of the formation of nanoelectrodes by gap formation using electromigration. This type of in-situ formation of a nanogap allows for a direct correlation of the electrical properties during the nanogap formation to the actual nanostructural changes that can be observed in the TEM. Recent examples of sub $1 \AA$ resolution in the temperature range from $100 \mathrm{~K}$ to $1000 \mathrm{~K}$ will be shown, in part in combination with electrical in-situ measurements of nanogaps formed by electromigration. 
Figure 1. Series of [110] HREM images taken from a movie. The black dots represent $\mathrm{Au}$ atom columns along the viewing direction. The time is indicated as insets on the left. In the middle three figures a dislocation can be observed in the top layer. Between 5.0 seconds and one image before the one of 8.5 seconds, no change is observed. The contrast of the series of images was enhanced by applying FFT filtering (reducing the contrast of features larger than $0.8 \mathrm{~nm}$ and smaller than $0.06 \mathrm{~nm}$ ). Ths movie was made with a Philips CM300UT microscope. Data taken with the FEI aberration corrected TEM show even more clearly the atom positions. The atom positions as deduced from these series of images fit very well with molecular dynamics simulations [1]

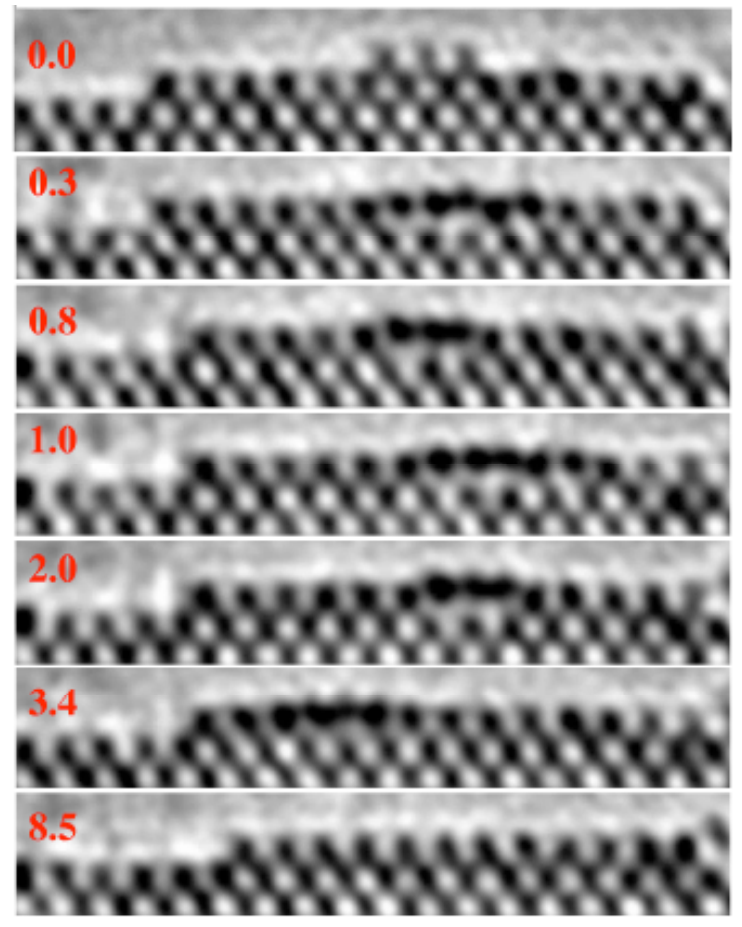

Figure 2. Evolution of a $7 \mathrm{~nm}$ gold particle in the quasi-molten state. The heater temperature is set to $415^{\circ} \mathrm{C}$ in figures (a) - (d) and $450^{\circ} \mathrm{C}$ in (e) and (f). (a) Initial decahedral structure, (b) rotated decahedral structure, (c) distorted icosahedral structure (d) cuboctahedral structure, (e) highly faceted Marks decahedral structure with 5-fold twin axis parallel to illumination.

Again Marks Decahedral structure with 5-fold axis inclined normal to the electron beam.

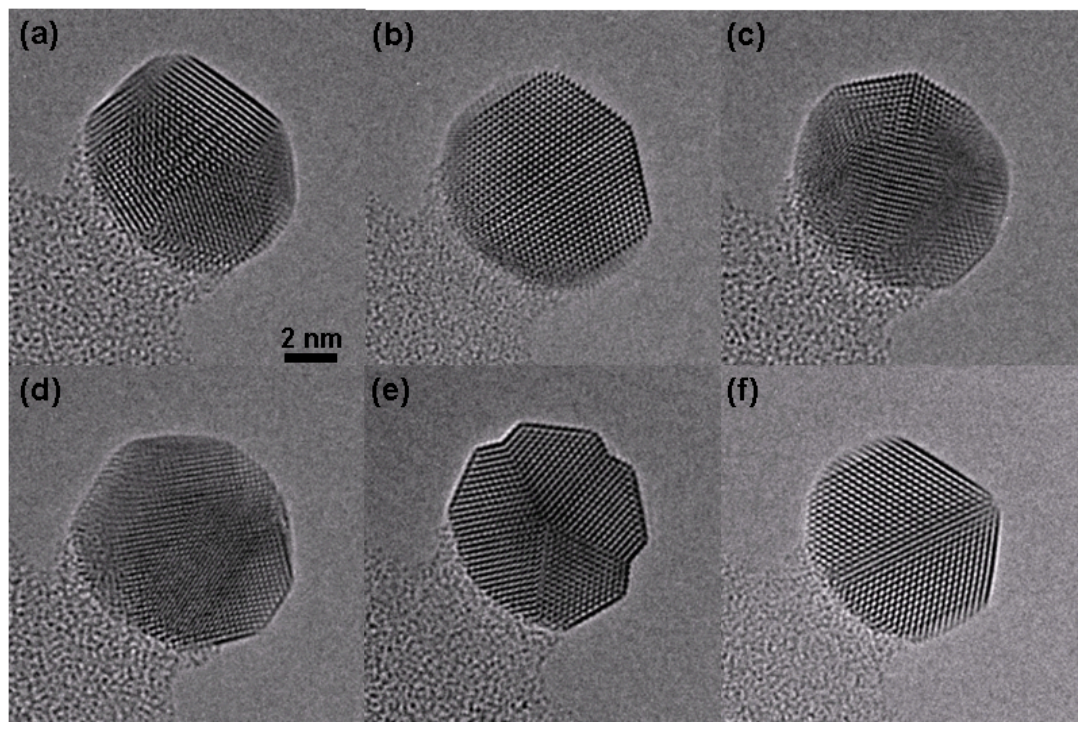

\section{References}

[1] Zandbergen, HW, Pao, L.Y \& Srolovitz, D.J. Phys. Rev. Lett. , 98, 036103-036106. (2007)..

[2] Huis, MA van, Young, NP, Pandraud, G, Creemer, JF, Vanmaekelbergh, D, Kirkland, A.I \& Zandbergen, HW. Advanced materials, 21, 4992-4995. (2009). 